\title{
An Efficient Image Processing Technique to Count Red Blood Cells
}

\author{
Joseph George ${ }^{1} \mathrm{~T}_{\text {Sobha }}{ }^{1}$ Arun Ashok V ${ }^{2}$ Jose Eldhose $^{2}$ \\ 1 Professors, Adi Shankara Institute of Engineering and Technology, Kalady, Kerala \\ 2 students, Adi Shankara Institute of Engineering and Technology, Kalady, Kerala
}

\begin{abstract}
Shortage of red blood cells (RBC), that constitutes 99 percent of blood cells and specialized as oxygen carrier, causes various blood disorders. The $\mathrm{RBC}$ is the important parameter while diagnosis and pathological study. Here, we create a system to detect to count the number of $\mathrm{RBC}$ in the blood smear image. The process is initiated by image acquisition and image enhancement where noise is removed from the images and the edges are preserved to converting to binary images thus separating the region of interest from the background. Further, by segmentation process we differentiate $\mathrm{RBC}$ from the various other components in the blood. Morphological operations are applied on the blood image followed by RBC counting using Hough transform which is an efficient image segmentation technique. The primary goal of the proposed system is to detect and count all the RBC including the overlapping one in the blood smear image.
\end{abstract}

Key Words: Image acquisition, Binarization, Morphological, Segmentation, Hough Transform

\section{INTRODUCTION}

Image Processing or Digital Image Processing is technique to enhance image quality by applying mathematical operations. Identification of two dimensional signal and enhancing it by comparing with standard signal is the important process of an Image Processing Projects. The second technique of image processing project is to switch characteristic parameters associated with digital images. This area has experienced continuous and significant expansion in recent years. The usefulness of this technology is clear in many various disciplines covering medicine through remote sensing. Easy of access of various advanced tools and hardware has further enhanced the usefulness of image processing. Recently various applications and contributions of new results and novel techniques from this important technology.

Digital image processing applications are widely used in the area of medical applications, restorations and enhancements, digital cinema, image transmission and coding, color processing, remote sensing, robot vision, hybrid techniques, facsimile, pattern recognition, registration techniques, multidimensional image processing image processing architectures and workstations, video processing, programmable DSPs for video high resolution display, high-quality color representation, super-highdefinition image processing, impact of standardization on image processing.

\subsection{Red Blood Cells}

Red blood cells (RBCs), also called erythrocytes, are the most common type of blood cell and the vertebrate organism's principal means of delivering oxygen $(\mathrm{O} 2)$ to the body tissues via the blood flow through the system. They take up oxygen in the lungs or gills and release it into tissues while squeezing through the body's capillaries. The cytoplasm of erythrocytes is rich in hemoglobin, an iron-containing biomolecule that can bind oxygen and is responsible for the red color of the cells. The cell membrane is composed of proteins and lipids, and this structure provides properties essential for physiological cell function such as deformability and stability while traversing the circulatory system and specifically the capillary network.

In humans, mature red blood cells are flexible and oval biconcave disks. They lack a cell nucleus and most organelles, in order to accommodate maximum space for hemoglobin. Approximately 2.4 million new erythrocytes are produced per second. The cells develop in the bone marrow and circulate for about 100120 days in the body before their components are recycled by macrophages. Each circulation takes about 20 seconds. Approximately a quarter of the cells in the human body are red blood cells. Red blood cells are also known as RBCs, red cells, red blood corpuscles, haematids, erythroid cells or packed red blood cells (pRBC) are red blood cells that have been donated, processed, and stored in a blood bank for blood transfusion.

\begin{tabular}{|c|c|c|}
\hline \multirow{2}{*}{ Blood Cell Type } & \multicolumn{2}{|c|}{ Mender } \\
\cline { 2 - 3 } & Men & Women \\
\hline RBC & $4.5-6.0$ million/microlitre & $4.0-5.0$ million/microlitre \\
\hline WBC & $4.5-11.0$ thousand/microlitre & $4.5-11.0$ thousand/microlitre \\
\hline Platelet & $150-450$ thousand/microlitre & $150-450$ thousand/microlitre \\
\hline Hematocrit & $42 \%$ to $50 \%$ & $36 \%$ to $45 \%$ \\
\hline Hemoglobin & $14-17$ grams $/ 100$ millitre & $12-15$ grams $/ 100$ millitre \\
\hline \multicolumn{3}{|c}{ Figure $1.1:$ Blood Count in humans }
\end{tabular}

\subsection{Blood diseases involving the Red Blood Cells}

Anemia is a diseases characterized by low oxygen transport capacity of the blood, due to low red cell count or some abnormality of the red blood cells or the hemoglobin. Sickle-cell disease may be a genetic disorder that leads to abnormal hemoglobin molecules. When these release their oxygen load in the tissues, they become insoluble, leading to misshaped red blood cells. These sickle shaped red cells 
are less deformable and viscoelastic meaning that they need become rigid and may cause vessel blockage, pain, strokes, and other tissue damage. Thalassemia may be a genetic disorder that leads to the assembly of an abnormal ratio of hemoglobin subunits. Spherocytosis may be a genetic disorder that causes a defect within the red blood cells cytoskeleton, causing the red blood cells to be small, sphere-shaped, and fragile rather than donut-shaped and versatile. Pernicious anemia is an autoimmune disorder wherein the body lacks factor, required to soak up vitamin B12 from food. Vitamin B12 is required for the assembly of hemoglobin.

\section{LITERATURE REVIEW}

Counting of RBC in a blood sample can give the pathologists valuable information regarding various hematological disorders. There are four steps involved in estimating the RBC. These are acquisition, segmentation, feature extraction and estimating. The acquisition step used the prevailing blood sample images. Next the segmentation and feature extraction is done by using a morphological technique in order to distinguish the RBC form background and other cells. The last step is estimating the amount the amount of RBC by using Hough Transform. This technique is able to detect and estimate the number of $\mathrm{RBC}$ by determining the centre point of the circle and then using IMDISTLINE. [1]

The paper presents an accurate, robust mechanism to work out the distribution of blood smear particles.[2] This work doesn't address issues like deformed RBC shapes (teardrops, crescents, needles, or a spread of other forms), infected RBCs and additional overlapping cells which may be found with certain sorts of diseases. Here, a set of blood Pap test images were taken to show that their proposed framework is more accurate as compared with some classical methods, and is also far more robust for degraded images which are blurry and/or noisy. First comparison method de-noises the image with a median type filter with a $3 \times 3$ mask function then uses Otsu binarization to make an intermediate image. Then a second intermediate image is made using canny edge detection. These two intermediate images are merged into the end image, objects with closed boundaries are filled with pixel wise, and the number of objects is counted using watershed segmentation. The second comparison method performs an equivalent procedure using Otsu binarization but skips the canny edge detection step. An image involves some unwanted particles (noise). Therefore, some pre-processing is required which is named image preparation phase. This phase consists of two steps which are histogram equalization and segmentation. In this step, histogram equalization, the intensity value of the given image is adjusted using image intensity transformation. In the second step, segmentation, blood corpuscle are detected by differentiate them from the background in terms of contrast. Changes in contrast can be detected by image processing operators that calculate the gradient of an image. [3]

Morphological image processing is a picture processing method that deals with recognition of an object, which was supported pre-defined criteria on their morphologies. Here, the acquired image is with a dimension of $640 \times 480$ pixels a 24-bit RGB JPG image. For the simple image binarization, the RGB image got to be grey-scaled image. The intensity remains kept to be proportional, with a 256 scale of shade from lightlessness to white. The produced grey-scaled image must be enhanced by using contrastlimited adaptive histogram equalization (CLHAE). The morphological operation to the RBCs is intended for recognizing RBCs, before the number of cells to be counted. At this stage, clumped cells which are commonly found in microscopy - need to be further separated. The watershed segmentation algorithm is then implemented to get clearer boundaries among clumped cells. This algorithm will perform task to determine the gradient magnitude, foreground markers and background markers. This algorithm is terminated by determination of watershed ridge lines. Region of Interest (ROI) is employed for determination of which groups of blood cells which can be separated. The adjoining RBCs can be recognized by determining the morphological properties of all related cells. The separation can then being supported parameter of cells diameter. Cells splitting process is accomplished by implemented algorithm, which may be ordered as follow. First the sides of the adjoining RBCs are going to be detected, which ends up the cells with two consecutive boundaries, i.e. inner and outer boundaries. The next step is devoted to remove these outer boundaries, and image of separated RBCs will be produced. [4]

\section{RESEARCH METHODOLOGY}

\subsection{Existing System}

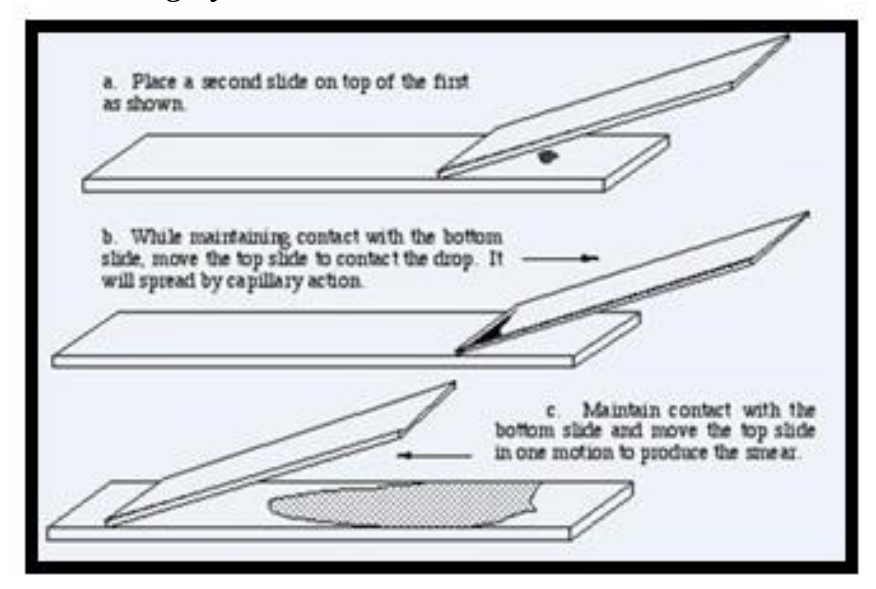

Figure 3.1 Existing method

Counting the cells during a patient's blood was performed manually, by viewing a slide prepared with a sample of the patient's blood under a microscope. Nowadays, this process is usually automated by use of an automatic analyzer. The Hemocytometer may be a device originally designed for the counting of blood cells. It is also used to count other sorts of cells as like as other microscopic particles. Hemocytometer consists of a thick glass slide with an oblong indentation that makes a chamber which is engraved with a laser-etched grid of perpendicular lines. The device is carefully crafted in order that the world bounded by the lines is understood and 
therefore the depth of the blood is well mixed even though not shaken and placed on a rack in the analyzer. Here, it has many different components to analyses different elements in the blood. Also, this cell counting component counts the numbers and kinds of various cells within the blood. The final results are printed or it may send to a computer for review.

\subsection{Drawbacks of Existing System}

Manual counting results in sampling error because so few cells are counted compared with automated analysis. Medical technologists examine blood film via a microscope for a few CBCs, not only to seek out abnormal white cells, but also because variation within the shape of red cells is an important diagnostic tool. Although automated analyzers give fast, reliable results regarding the amount, average size, and variation in size of red blood cells, they are doing not detect cells shapes. Also, some normal patient's platelets will clump in anti-coagulated blood, which causes automatic analyses to offer a falsely low platelet count. The technician viewing the slide in these cases will see clumps of platelets and may estimate if there are low, normal, or high numbers of platelets.

\section{DESIGN AND MATERIALS}

\subsection{System Architecture}

\subsubsection{Image Acquisition}

In data acquisition, we used sample images from an online medical library. These images can be acquired by the user through a simple user interface, from their computers. Here, these images contain noise and are not fit for direct processing they need to be enhanced for further analysis. Figure shows the block diagram of the red blood cells counting process.

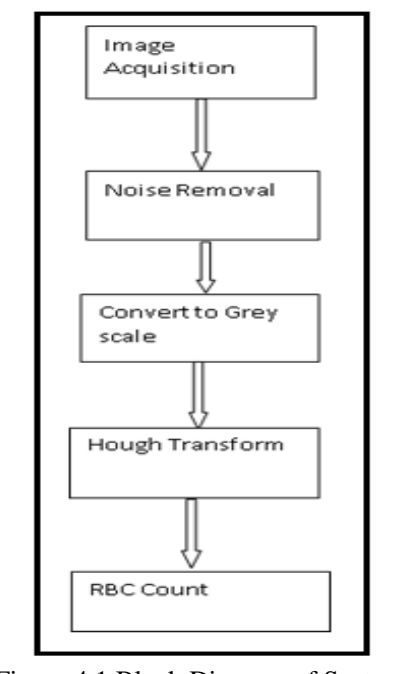

Figure 4.1 Block Diagram of System

\subsubsection{RBC Count Algorithm}

Step 1: Start

Step 2: If file is selected in image acquisition, go to step 4.

Step 3: If file is not selected, go to step 9.

Step 4: Noise removal will be performed.
Step 5: Edge Detection method by canny edge detection.

Step 6: Morphological operation will be performed.

Step 7: RBC size extraction.

Step 8: RBC will be counted i.e. RBC count.

Step 9: End

\subsection{Hardware and Software Tools}

\section{Hardware Tools}

- Processor: Intel or AMD x86 processor which supports SSE2 instruction set.

- Disk Space: 1 GB for MATLAB only, 3 GB for a typical installation.

- $\quad$ RAM: At least $2048 \mathrm{MB}$ recommended

\section{Software Tools}

- Windows 7 or Service Pack 1

- Windows Vista Service Pack 2

- Windows XP Service Pack 3

- Windows XP x64 Edition Service Pack 2

- Windows Server 2008 Service Pack 2 or R2

- Windows Server 2003 R2 Service Pack2

MATLAB:

- MATLAB version used while developing the system will be 2011a released on 8th April, 2011.The toolkits used will be:

- Image processing toolkit.

- Signal processing toolkit.

\section{CONCLUSIONS}

This study successfully uses various image processing techniques for Red Blood Cell Estimation. It utilizes morphological approaches for segmentation, extraction and estimation so as to unravel problem in image processing of the red blood cells. The results of the image act as an accurate outcome of determining the amount of red blood cells by using Hough transform technique. It proposes a picture processing system that uses MATLAB software for blood corpuscle counting. By using the MATLAB all the importance aspects of a correct algorithm has been successfully produced. With a correct algorithm, the red blood cells can be detected and segmented as well as estimate the number of the red blood cells. It enables the study of the morphological features of RBC by the pathologist can determine whether the person is normal referring the amount of RBC in human blood. Here, actual volume of the blood sample is calculated with proper magnification factor. There is a requirement for fast and cost-effective production of blood corpuscle count reports. This system includes an efficient and efficient method in recognizing and counting blood corpuscle $s$ as a practical alternative to the manual blood cell counting. Since it's a non-going study, more enhancements and improvement could be done in the future works. The system can be further improvised for detecting various diseases related to different blood cell morphologies. 


\section{REFERENCES}

[1] "Red Blood Cells Estimation Using Hough Transform Technique", Signal Image Processing; Nasrul Humaimi Mahmood and Muhammad Asraf Mansor. An International Journal (SIPIJ Vol.3,) No 2 April 2012.

[2] "Counting of RBCs and WBCs in noisy normal blood smear microscopic im-ages"; M.Habibzadeh, A. Krzyak, T. Fevens, A. Sadr, Proc. of SPIE Vol. 7963 79633I-1,05 July 2011.

[3] "Automated Red Blood Cell Counting"; Alaa Hamouda, Ahmed Y. Khedr, and Rabie A. Ramadan, INTERNATIONAL JOURNAL OF COMPUTING SCIENCE, VOL. 1, NO. 2, FEBRUARY, 2012.

[4] "Automated morphological processing for counting the number of red blood cell” A M T Nasution, E K Suryaningtyas, Proceeding of the 2008 International Joint Conference in Engineering IJSE2008, Jakarta, Indonesia, August 4-5. 